\title{
Information Sharing Across Private Databases
}

\author{
Rakesh Agrawal \\ Alexandre Evfimievski * \\ Ramakrishnan Srikant \\ IBM Almaden Research Center \\ 650 Harry Road, San Jose, CA 95120
}

\begin{abstract}
Literature on information integration across databases tacitly assumes that the data in each database can be revealed to the other databases. However, there is an increasing need for sharing information across autonomous entities in such a way that no information apart from the answer to the query is revealed. We formalize the notion of minimal information sharing across private databases, and develop protocols for intersection, equijoin, intersection size, and equijoin size. We also show how new applications can be built using the proposed protocols.
\end{abstract}

\section{INTRODUCTION}

Information integration has long been an area of active database research $[12,16,21,27,48]$. So far, this literature has tacitly assumed that the information in each database can be freely shared. However, there is now an increasing need for computing queries across databases belonging to autonomous entities in such a way that no more information than necessary is revealed from each database to the other databases. This need is driven by several trends:

- End-to-end Integration: E-business on demand requires end-to-end integration of information systems, from the supply chain to the customer-facing systems. This integration occurs across autonomous enterprises, so full disclosure of information in each database is undesirable.

- Outsourcing: Enterprises are outsourcing tasks that are not part of their core competency. They need to integrate their database systems for purposes such as inventory control.

- Simultaneously compete and cooperate: It is becoming common for enterprises to cooperate in certain areas and compete in others, which requires selective information sharing.

- Security: Government agencies need to share information for devising effective security measures, both within the same government and across governments. However, an

*Currently at Cornell University, Ithaca, NY 14853.

Permission to make digital or hard copies of all or part of this work for personal or classroom use is granted without fee provided that copies are not made or distributed for profit or commercial advantage and that copies bear this notice and the full citation on the first page. To copy otherwise, to republish, to post on servers or to redistribute to lists, requires prior specific permission and/or a fee.

SIGMOD 2003, June 9-12, 2003, San Diego, CA

Copyright 2003 ACM 1-58113-634-X/03/06 ...\$5.00. agency cannot indiscriminately open up its database to all other agencies.

- Privacy: Privacy legislation and stated privacy policies place limits on information sharing. However, it is still desirable to mine across databases while respecting privacy limits.

We propose a new paradigm of minimal necessary information sharing across private databases. Intuitively, given a database query spanning multiple private databases, we wish to compute the answer to the query without revealing any additional information apart from the query result. We will sometimes relax this constraint to allow some minimal additional information to be revealed.

\subsection{Motivating Applications}

We give two prototypical applications to make the above paradigm concrete.

Application 1: Selective Document Sharing Enterprise $R$ is shopping for technology and wishes to find out if enterprise $S$ has some intellectual property it might want to license. However, $R$ would not like to reveal its complete technology shopping list, nor would $S$ like to reveal all its unpublished intellectual property. Rather, they would like to first find the specific technologies for which there is a match, and then reveal information only about those technologies. This problem can be abstracted as follows.

We have two databases $D_{R}$ and $D_{S}$, where each database contains a set of documents. The documents have been preprocessed to only include the most significant words, using some measure such as term frequency times inverse document frequency [41]. We wish to find all pairs of similar documents $d_{R} \in D_{R}$ and $d_{S} \in D_{S}$, without revealing the other documents. In database terminology, we want to compute the join of $D_{R}$ and $D_{S}$ using the join predicate $f\left(\left|d_{R} \cap d_{S}\right|,\left|d_{R}\right|,\left|d_{S}\right|\right)>\tau$, for some similarity function $f$ and threshold $\tau$. The function $f$ could be $\left|d_{R} \cap d_{S}\right| /\left(\left|d_{R}\right|+\left|d_{S}\right|\right)$, for instance.

Many applications map to this abstraction. For example, two government agencies may want to share documents, but only on a need-to-know basis. They would like to find similar documents contained in their repositories in order to initiate their exchange.

Application 2: Medical Research Imagine a future where many people have their DNA sequenced. A medical researcher wants to validate a hypothesis connecting a DNA sequence $D$ with a reaction to drug $G$. People who have taken the drug are partitioned into four groups, based on whether or not they had an adverse reaction and whether or not their DNA contained the specific sequence; the researcher needs the number of people in each group. DNA sequences and medical histories are stored in databases in autonomous enterprises. Due to privacy concerns, the enterprises do not wish to provide any information about an individual's DNA sequence or medical history, but still wish to help with the research. 
Assume that the table $T_{R}$ (person_id, pattern) stores whether a person's DNA contains pattern $D$ and $T_{S}$ (person_id, drug, reaction) captures whether a person took drug $G$ and whether the person had an adverse reaction. $T_{R}$ and $T_{S}$ belong to two different enterprises. The researcher wants to get the answer to the following query.



We want the property that the researcher should get to know the counts and nothing else, and the enterprises should not learn any new information about any individual.

\subsection{Current Techniques}

We discuss next some existing techniques that one might use for building the above applications, and why they are inadequate.

- Trusted Third Party: The main parties give the data to a "trusted" third party and have the third party do the computation $[7,30]$. However, the third party has to be completely trusted, both with respect to intent and competence against security breaches. The level of trust required is too high for this solution to be acceptable.

- Secure Multi-Party Computation: Given two parties with inputs $x$ and $y$ respectively, the goal of secure multi-party computation is to compute a function $f(x, y)$ such that the two parties learn only $f(x, y)$, and nothing else. See [26, 34] for a discussion of various approaches to this problem.

Yao [49] showed that any multi-party computation can be solved by building a combinatorial circuit, and simulating that circuit. A variant of Yao's protocol is presented in [37] where the number of oblivious transfers is proportional to the number of inputs and not the size of the circuit. We show in Appendix A that our specialized algorithms are substantially faster than using a circuit, and in particular, the communication costs for circuits make them impractical for our problems.

\subsection{Paper Outline}

The rest of the paper is organized as follows. We formally state the problem and the scope of this paper in Section 2. We develop the protocol for computing the intersection of two sets in Section 3, and extend this protocol for equijoins in Section 4. We describe the protocols for intersection size and equijoin size in Section 5. In Section 6, we give a cost analysis of these protocols, and use this analysis to estimate the execution times of the application examples above. We conclude with a summary and directions for future work in Section 7.

\section{MINIMAL INFORMATION SHARING}

\subsection{Security Model}

We develop our solutions in a setting in which there is no third party [26]. The main parties directly execute a protocol, which is designed to guarantee that they do not learn any more than they would have learnt had they given the data to a trusted third party and got back the answer.

We assume honest-but-curious behavior [26]. The parties follow the protocol properly with the exception that they may keep a record of all the intermediate computations and received messages, and analyze the messages to try to learn additional information.

\begin{tabular}{|l|l|l|}
\hline \multicolumn{3}{|c|}{ Cryptographic Protocol } \\
\hline $\begin{array}{l}\text { Secure } \\
\text { Commu- } \\
\text { nication }\end{array}$ & $\begin{array}{l}\text { Libraries } \\
\text { (including } \\
\text { encryption } \\
\text { primitives) }\end{array}$ & Database \\
\hline \multicolumn{3}{|c|}{ Operating System } \\
\hline
\end{tabular}

Figure 1: System Components

This behavior is also referred to as semi-honest or passive behavior.

Figure 1 shows the different components required for building a system for information integration with minimal sharing. Our focus will be on the cryptographic protocol. We assume the use of standard libraries or packages for secure communication and encryption primitives.

\subsection{Problem Statement}

We now formally state the problem we study in this paper.

Problem Statement (Ideal) Let there be two parties $R$ (receiver) and $S$ (sender) with databases $D_{R}$ and $D_{S}$ respectively. Given a database query $Q$ spanning the tables in $D_{R}$ and $D_{S}$, compute the answer to $Q$ and return it to $R$ without revealing any additional information to either party.

Problem Statement (Minimal Sharing) Let there be two parties $R$ and $S$ with databases $D_{R}$ and $D_{S}$ respectively. Given a database query $Q$ spanning the tables in $D_{R}$ and $D_{S}$, and some categories of information $I$, compute the answer to $Q$ and return it to $R$ without revealing any additional information to either party except for information contained in $I$.

For example, if the query $Q$ is a join $T_{R} \bowtie T_{S}$ over two tables $T_{R}$ and $T_{S}$, the additional information $I$ might be the number of records in each table: $\left|T_{R}\right|$ and $\left|T_{S}\right|$. Note that whatever $R$ can infer from knowing the answer to the query $Q$ and the additional information $I$ is fair game. For instance, if the query $Q$ is an intersection $V_{S} \cap V_{R}$ between two sets $V_{S}$ and $V_{R}$, then for all $v \in\left(V_{R}-\left(V_{S} \cap V_{R}\right)\right), R$ knows that these values were not in $V_{S}$.

We assume that the query $Q$ is revealed to both parties. One can think of other applications where the format of $Q$ is revealed, but not the parameters of $Q$ (e.g., in private information retrieval, discussed in Section 2.4).

\subsubsection{Operations}

In this paper, we focus on four operations: intersection, equijoin, intersection size, and equijoin size.

Let $S$ have a database table $T_{S}$, and $R$ have a table $T_{R}$, with both tables having a specific attribute $A$ in their schemas. The attribute $A$ takes its values from a given set $V$. Let $V_{S}$ be the set of values (without duplicates) that occur in $T_{S} . A$, and let $V_{R}$ be the set of values occurring in $T_{R}$. $A$. For each $v \in V_{S}$, let $\operatorname{ext}(v)$ be all records in $T_{S}$ where $T_{S} . A=v$, i.e., $\operatorname{ext}(v)$ is the extra information in $T_{S}$ pertaining to $v$. We show how to compute three kinds of queries over $T_{S}$ and $T_{R}$ :

- Intersection: Party $R$ learns the set $V_{S} \cap V_{R}$, the value $\left|V_{S}\right|$, and nothing else; party $S$ learns $\left|V_{R}\right|$ and nothing else (Section 3). 
- Equijoin: Party $R$ learns $V_{S} \cap V_{R}$, ext $(v)$ for all $v \in V_{S} \cap V_{R}$, $\left|V_{S}\right|$, and nothing else; party $S$ learns $\left|V_{R}\right|$ and nothing else (Section 4).

- Intersection Size: Party $R$ learns the values of $\left|V_{S} \cap V_{R}\right|$, $\left|V_{S}\right|$, and nothing else; party $S$ learns $\left|V_{R}\right|$ and nothing else (Section 5).

Thus in the terminology of our problem statement above, the query $Q$ for the three problems corresponds to $V_{S} \cap V_{R}, T_{S} \bowtie T_{R}$ (with $\operatorname{ext}(v)$ used to compute the join), and $\left|V_{S} \cap V_{R}\right|$ respectively. In all three cases, the additional information $I$ consists of $\left|V_{R}\right|$ and $\left|V_{S}\right|$.

We also extend the intersection size protocol to obtain an equijoin size protocol that computes $\left|T_{S} \bowtie T_{R}\right|$ (Section 5.2). However, $R$ learns $\left|V_{S}\right|$, the distribution of duplicates in $T_{S} . A$, and based on the distribution of duplicates, some subset of information in $V_{S} \cap V_{R} . S$ learns $\left|V_{R}\right|$ and the distribution of duplicates in $T_{R} . A$.

\subsection{Limitations}

Multiple Queries While we provide guarantees on how much the parties learn from a single query, our techniques do not address the question of what the parties might learn by combining the results of multiple queries. The first line of defence against this problem is the scrutiny of the queries by the parties. In addition, query restriction techniques from the statistical database literature $[1,44]$ can also help. These techniques include restricting the size of query results [17, 23], controlling the overlap among successive queries [19], and keeping audit trails of all answered queries to detect possible compromises [13].

Schema Discovery and Heterogeneity We do not address the question of how to find which database contains which tables and what the attribute names are; we assume that the database schemas are known. We also do not address issues of schema heterogeneity. See $[21,29]$ and references therein for some approaches to these problems.

\subsection{Related Work}

In [35], the authors consider the problem of finding the intersection of two lists while revealing only the intersection. They present two solutions: the first involves oblivious evaluations of $n$ polynomials of degree $n$ each, where $n$ is the number of elements in the list; the second solution requires oblivious evaluation of $n^{2}$ linear polynomials. In the context of databases, $n$ will be quite large. In [28], the authors consider the problem of finding people with common preferences, without revealing the preferences. They give intersection protocols that are similar to ours, but do not provide proofs of security.

In the problem of private information retrieval $[11,14,15,32$, 45], the receiver $R$ obtains the $i$ th record from set of $n$ records held by the sender $S$ without revealing $i$ to $S$. With the additional restriction that $R$ should only learn the value of one record, the problem becomes that of symmetric private information retrieval [25]. This literature will be useful for developing protocols for the selection operation in our setting.

The problem of privacy-preserving data mining is also related. The randomization approach $[6,22,40]$ focuses on individual privacy rather than on database privacy, and reveals randomized information about each record in exchange for not having to reveal the original records to anyone. More closely related is the work in [33] on building a decision-tree classifier across multiple databases, without revealing the individual records in each database to the other databases. Algorithms for mining associations rules across multiple databases have been described in [31] and [47] for hori- zontally and vertically partitioned data respectively.

The context for the work presented in this paper is our effort to design information systems that protect the privacy and ownership of individual information while not impeding the flow of information. Our other related papers include $[2,3,4,5]$.

\section{INTERSECTION}

\subsection{A Simple, but Incorrect, Protocol}

A straightforward idea for computing the intersection $V_{S} \cap V_{R}$ would be to use one-way hash functions [38]. Here is a simple protocol that appears to work:

1. Both $S$ and $R$ apply hash function $h$ to their sets, yielding $X_{S}=h\left(V_{S}\right)=\left\{h(v) \mid v \in V_{S}\right\}$ and $X_{R}=h\left(V_{R}\right)=\left\{h(v) \mid v \in V_{R}\right\}$.

2. $S$ sends its hashed set $X_{S}$ to $R$.

3. $R$ sets aside all $v \in V_{R}$ for which $h(v) \in X_{S}$; these values form the set $V_{S} \cap V_{R}$.

Unfortunately, $R$ can learn a lot more about $V_{S}$ (with honestbut-curious behavior). For any arbitrary value $v \in V-\left(V_{S} \cap V_{R}\right)$, $R$ can simply compute $h(v)$ and check whether $h(v) \in X_{S}$ to determine whether or not $v \in V_{S}$. In fact, if the domain $V$ is small, $R$ can exhaustively go over all possible values and completely learn $V_{S}$.

The intersection protocol we propose next fixes the deficiencies of this protocol.

\subsection{Building Blocks}

We first describe two building blocks used in the proposed protocols.

\subsubsection{Commutative Encryption}

Our definition of commutative encryption below is similar to the constructions used in $[9,18,20,42]$ and others. Informally, a commutative encryption is a pair of encryption functions $f$ and $g$ such that $f(g(v))=g(f(v))$. Thus by using the combination $f(g(v))$ to encrypt $v$, we can ensure that $R$ cannot compute the encryption of a value without the help of $S$. In addition, even though the encryption is a combination of two functions, each party can apply their function first and still get the same result.

Definition 1 (Indistinguishability). Let $\Omega_{k} \subseteq\{0,1\}^{k}$ be a finite domain of $k$-bit numbers. Let $\mathcal{D}_{1}=\mathcal{D}_{1}\left(\bar{\Omega}_{k}\right)$ and $\mathcal{D}_{2}=\mathcal{D}_{2}\left(\Omega_{k}\right)$ be distributions over $\Omega_{k}$. Let $\mathcal{A}_{k}(x)$ be an algorithm that, given $x \in \Omega_{k}$, returns either true or false. We define distribution $\mathcal{D}_{1}$ of random variable $x \in \Omega_{k}$ to be computationally indistinguishable from distribution $\mathcal{D}_{2}$ if for any family of polynomial-step (w.r.t. $k$ ) algorithms $\mathcal{A}_{k}(x)$, any polynomial $p(k)$, and all sufficiently large $k$

$$
\operatorname{Pr}\left[\mathcal{A}_{k}(x) \mid x \sim \mathcal{D}_{1}\right]-\operatorname{Pr}\left[\mathcal{A}_{k}(x) \mid x \sim \mathcal{D}_{2}\right]<\frac{1}{p(k)}
$$

where $x \sim \mathcal{D}$ denotes that $x$ is distributed according to $\mathcal{D}$, and $\operatorname{Pr}\left[\mathcal{A}_{k}(x)\right]$ is the probability that $\mathcal{A}_{k}(x)$ returns true.

Throughout this paper, we will use "indistinguishable" as shorthand for "computationally indistinguishable".

DEFINITION 2 (COMMUTATIVE ENCRYPTION). A commutative encryption $\mathcal{F}$ is a computable (in polynomial time) function $f: \operatorname{Key} \mathcal{F} \times \operatorname{Dom} \mathcal{F} \rightarrow \operatorname{Dom} \mathcal{F}$, defined on finite computable domains, that satisfies all properties listed below. We denote $f_{e}(x) \equiv$ $f(e, x)$, and use " $\in_{r}$ " to mean "is chosen uniformly at random from". 
1. Commutativity: For all $e, e^{\prime} \in \mathrm{Key} \mathcal{F}$ we have

$$
f_{e} \circ f_{e^{\prime}}=f_{e^{\prime}} \circ f_{e} \text {. }
$$

2. Each $f_{e}: \operatorname{Dom} \mathcal{F} \rightarrow \operatorname{Dom} \mathcal{F}$ is a bijection.

3. The inverse $f_{e}^{-1}$ is also computable in polynomial time given e.

4. The distribution of $\left\langle x, f_{e}(x), y, f_{e}(y)\right\rangle$ is indistinguishable from the distribution of $\left\langle x, f_{e}(x), y, z\right\rangle$, where $x, y, z \in_{r}$ $\operatorname{Dom} \mathcal{F}$ and $e \in_{r}$ Key $\mathcal{F}$.

Informally, Property 1 says that when we compositely encrypt with two different keys, the result is the same irrespective of the order of encryption. Property 2 says that two different values will never have the same encrypted value. Property 3 says that given an encrypted value $f_{e}(x)$ and the encryption key $e$, we can find $x$ in polynomial time. ${ }^{1}$ Property 4 says that given a value $x$ and its encryption $f_{e}(x)$ (but not the key $e$ ), for a new value $y$, we cannot distinguish between $f_{e}(y)$ and a random value $z$ in polynomial time. Thus we can neither encrypt $y$ nor decrypt $f_{e}(y)$ in polynomial time. Note that this property holds only if $x$ is a random value from $\operatorname{Dom} \mathcal{F}$, i.e., the adversary does not control the choice of $x$.

Example 1 Let Dom $\mathcal{F}$ be all quadratic residues modulo $p$, where $p$ is a "safe" prime number, i.e., both $p$ and $q=(p-1) / 2$ are primes. Let Key $\mathcal{F}$ be $\{1,2, \ldots, q-1\}$. Then, assuming the Decisional Diffie-Hellman hypothesis (DDH) [10], the power function

$$
f_{e}(x) \equiv x^{e} \bmod p
$$

is a commutative encryption:

- The powers commute: $\left(x^{d} \bmod p\right)^{e} \bmod p=x^{d e} \bmod p=\left(x^{e} \bmod p\right)^{d} \bmod p$.

- Each of the powers $f_{e}$ is a bijection with its inverse being $f_{e}^{-1}=f_{e^{-1} \bmod q}$.

- DDH claims that for any generating $(\neq 1)$ element $g \in$ Dom $\mathcal{F}$ the distribution of $\left\langle g^{a}, g^{b}, g^{a b}\right\rangle$ is indistinguishable from the distribution of $\left\langle g^{a}, g^{b}, g^{c}\right\rangle$, where $a, b, c \in_{r}$ Key $\mathcal{F}$. A 3tuple $\left\langle g^{a}, g^{b}, z\right\rangle$ from the DDH can be reduced to our 4tuple $\left\langle x, x^{e}, y, z\right\rangle$ by taking $d \in_{r}$ Key $\mathcal{F}$ and making tuple $\left\langle g^{d},\left(g^{a}\right)^{d}, g^{b}, z\right\rangle$. Now a plays the role of $e, g^{d}$ of $x$, and $g^{b}$ of $y$; we test whether $z=\left(g^{b}\right)^{a}$ or is random. Thus, given DDH, $\left\langle x, x^{e}, y, y^{e}\right\rangle$ and $\left\langle x, x^{e}, y, z\right\rangle$ are also indistinguishable.

\subsubsection{Hash Function}

Besides a commutative encryption $\mathcal{F}$, we need a hash function to encode the values $v \in V$ into $x \in \operatorname{Dom} \mathcal{F}$. The hashes of values should not collide and should "look random," i.e., there should be no dependency between them that could help encrypt or decrypt one hashed value given the encryption of another. Since we apply commutative encryption to the hashed values $h(v)$ instead of $v$, the input for the encryption function will appear random, and we will be able to use Property 4 of commutative encryption to prove that our protocols are secure.

In the proofs of our security statements we shall rely on the standard random oracle model $[8,24,46]$. We assume that our hash function $h: V \rightarrow \operatorname{Dom} \mathcal{F}$ is ideal, which means that $h(v)$ can be considered computed by a random oracle: every time $h(v)$ is evaluated for a new $v \in V$, an independent random $x \in_{r} \operatorname{Dom} \mathcal{F}$ is chosen for $x=h(v)$.

\footnotetext{
${ }^{1}$ We only need this property for the join protocol, not for the intersection protocol.
}

We assume also that $|\operatorname{Dom} \mathcal{F}|$ is so large compared to $\left|V_{S} \cup V_{R}\right|$ that the probability of a collision is exponentially small. Let $N=$ $|\operatorname{Dom} \mathcal{F}|$; in the random oracle model, the probability that $n$ hash values have at least one collision equals [46]:

$$
\operatorname{Pr}[\text { collision }]=1-\prod_{i=1}^{n-1} \frac{N-i}{N} \approx 1-\exp \left(\frac{-n(n-1)}{2 N}\right)
$$

With 1024-bit hash values, half of which are quadratic residues, we have $N \approx 2^{1024} / 2 \approx 10^{307}$, and for $n=1$ million

$$
\operatorname{Pr}[\text { collision }] \approx 1-\exp \left(-\frac{10^{12}}{10^{307}}\right) \approx \frac{10^{12}}{10^{307}}=10^{-295} .
$$

For real-life hash functions, a collision within $V_{S}$ or $V_{R}$ can be detected by the server at the start of each protocol by sorting the hashes. If there is a collision between $v \in V_{S}$ and $v^{\prime} \in V_{R}$, it will cause inclusion of $v^{\prime}$ into the join (or intersection) by $R$ and the disclosure to $R$ of $S$ 's records containing $v .^{2}$

\subsection{Intersection Protocol}

Our proposed intersection protocol is as follows.

1. Both $S$ and $R$ apply hash function $h$ to their sets: $X_{S}=h\left(V_{S}\right)$ and $X_{R}=h\left(V_{R}\right)$. Each party randomly chooses a secret key: $e_{S} \in_{r}$ Key $\mathcal{F}$ for $S$ and $e_{R} \in_{r}$ Key $\mathcal{F}$ for $R$.

2. Both parties encrypt their hashed sets: $Y_{S}=f_{e_{S}}\left(X_{S}\right)=f_{e_{S}}\left(h\left(V_{S}\right)\right)$ and $Y_{R}=f_{e_{R}}\left(X_{R}\right)=f_{e_{R}}\left(h\left(V_{R}\right)\right)$

3. $R$ sends to $S$ its encrypted set $Y_{R}=f_{e_{R}}\left(h\left(V_{R}\right)\right)$, reordered lexicographically. ${ }^{3}$

4. (a) $S$ ships to $R$ its set $Y_{S}=f_{e_{S}}\left(h\left(V_{S}\right)\right)$, reordered lexicographically.

(b) $S$ encrypts each $y \in Y_{R}$ with $S$ 's key $e_{S}$ and sends back to $R$ pairs $\left\langle y, f_{e_{S}}(y)\right\rangle=\left\langle f_{e_{R}}(h(v)), f_{e_{S}}\left(f_{e_{R}}(h(v))\right)\right\rangle$.

5. $R$ encrypts each $y \in Y_{S}$ with $e_{R}$, obtaining $Z_{S}=f_{e_{R}}\left(f_{e_{S}}\left(h\left(V_{S}\right)\right)\right)$.

Also, from pairs $\left\langle f_{e_{R}}(h(v)), f_{e_{S}}\left(f_{e_{R}}(h(v))\right)\right\rangle$ obtained in Step 4(b) for $v \in V_{R}$, it creates pairs $\left\langle v, f_{e_{S}}\left(f_{e_{R}}(h(v))\right)\right\rangle$ by replacing $f_{e_{R}}(h(v))$ with the corresponding $v$.

6. $R$ selects all $v \in V_{R}$ for which $\left(f_{e_{S}}\left(f_{e_{R}}(h(v))\right) \in Z_{S}\right.$; these values form the set $V_{S} \cap V_{R}$.

\subsection{Proofs of Correctness and Security}

StATEMENT 1. Assuming there are no hash collisions, $S$ learns the size $\left|V_{R}\right|$ and $R$ learns the size $\left|V_{S}\right|$ and the set $V_{S} \cap V_{R}$.

PRoOF. By definition, $f_{e_{S}}$ and $f_{e_{R}}$ commute and are bijective. Assuming that hash function $h$ has no collisions on $V_{S} \cup V_{R}$,

$$
v \in V_{S} \cap V_{R} \text { iff } v \in V_{R} \text { and }\left(f_{e_{S}} \circ f_{e_{R}}\right)(h(v)) \in Z_{S},
$$

which means that $R$ does recover the correct set $V_{S} \cap V_{R}$. Both parties also learn the sizes $\left|V_{R}\right|$ and $\left|V_{S}\right|$, since $\left|V_{R}\right|=\left|Y_{R}\right|$ and $\left|V_{S}\right|=\left|Y_{S}\right|$.

\footnotetext{
${ }^{2}$ For the join protocol (Section 4$), R$ can check whether there was a collision between $v \in V_{S}$ and $v^{\prime} \in V_{R}$ by having $S$ include the value $v$ in $\operatorname{ext}(v)$.

${ }^{3}$ If we did not reorder and instead sent the values in the same order as the values in $V_{R}$, significant additional information could be revealed.
} 
Next we prove that, assuming the parties follow the protocol correctly, they learn nothing else about the other's sets. We first show that even given

$$
\left(\begin{array}{ccc}
x_{1} & \cdots & x_{m} \\
f_{e}\left(x_{1}\right) & \cdots & f_{e}\left(x_{m}\right)
\end{array}\right) \text { and } x_{m+1}
$$

there is no polynomial-time algorithm that can determine whether or not a value $u$ is in fact $f_{e}\left(x_{m+1}\right)$.

LEMMA 1. For polynomial $m$, the distribution of the $2 \times m$-tuple

$$
\left(\begin{array}{cccc}
x_{1} & \cdots & x_{m-1} & x_{m} \\
f_{e}\left(x_{1}\right) & \cdots & f_{e}\left(x_{m-1}\right) & f_{e}\left(x_{m}\right)
\end{array}\right)
$$

is indistinguishable from the distribution of the tuple

$$
\left(\begin{array}{cccc}
x_{1} & \ldots & x_{m-1} & x_{m} \\
f_{e}\left(x_{1}\right) & \cdots & f_{e}\left(x_{m-1}\right) & z_{m}
\end{array}\right),
$$

where $\forall i: x_{i} \in_{r} \operatorname{Dom} \mathcal{F}, z_{m} \in_{r} \operatorname{Dom} \mathcal{F}$, and $e \in_{r}$ Key $\mathcal{F}$.

PRoOf. Let us denote the distribution of the upper tuple by $\mathcal{D}_{m}$, and the distribution of the lower tuple by $\mathcal{D}_{m-1}$. If $\mathcal{D}_{m}$ and $\mathcal{D}_{m-1}$ are distinguishable by some polynomial algorithm $\mathcal{A}$, then $\left\langle x, f_{e}(x), y, f_{e}(y)\right\rangle$ and $\left\langle x, f_{e}(x), y, z\right\rangle$ from Property 4 of commutative encryption are also distinguishable by the following algorithm that takes $\left\langle x, f_{e}(x), y, u\right\rangle$ as argument:

1. For $i=1 \ldots m-1$, let $x_{i}=f_{e_{i}}(x)$ and $z_{i}=f_{e_{i}}\left(f_{e}(x)\right)$, where $e_{i} \in_{r} \operatorname{Key} \mathcal{F}$;

2. Let $x_{m}=y$ and $z_{m}=u$;

3. Submit tuple

$$
\left(\begin{array}{lll}
x_{1} & \ldots & x_{m} \\
z_{1} & \ldots & z_{m}
\end{array}\right)
$$

to algorithm $\mathcal{A}$ and output whatever it outputs.

For $i=1 \ldots m-1$, we have

$$
z_{i}=f_{e_{i}}\left(f_{e}(x)\right)=f_{e}\left(f_{e_{i}}(x)\right)=f_{e}\left(x_{i}\right),
$$

and all $x_{i}$ are indistinguishable from uniformly random (from Property 4 of commutative encryption). Therefore the distribution of the tuple given to $\mathcal{A}$ is indistinguishable from $\mathcal{D}_{m}$ when $\left\langle x, f_{e}(x), y, u\right\rangle$ is distributed as $\left\langle x, f_{e}(x), y, f_{e}(y)\right\rangle$, and from $\mathcal{D}_{m-1}$ when $\left\langle x, f_{e}(x), y, u\right\rangle$ is distributed as $\left\langle x, f_{e}(x), y, z\right\rangle$. So the assumption that $\mathcal{D}_{m}$ and $\mathcal{D}_{m-1}$ are distinguishable leads to the contradiction that Property 4 does not hold.

LEMMA 2. For polynomial $m$ and $n$, the distribution of the $2 \times n$-tuple

$$
\left(\begin{array}{cccccc}
x_{1} & \ldots & x_{m} & x_{m+1} & \ldots & x_{n} \\
f_{e}\left(x_{1}\right) & \ldots & f_{e}\left(x_{m}\right) & f_{e}\left(x_{m+1}\right) & \ldots & f_{e}\left(x_{n}\right)
\end{array}\right)
$$

is indistinguishable from the distribution of the tuple

$$
\left(\begin{array}{cccccc}
x_{1} & \ldots & x_{m} & x_{m+1} & \ldots & x_{n} \\
f_{e}\left(x_{1}\right) & \ldots & f_{e}\left(x_{m}\right) & z_{m+1} & \ldots & z_{n}
\end{array}\right),
$$

where $0 \leqslant m \leqslant n, \forall i: x_{i}, z_{i} \in_{r} \operatorname{Dom} \mathcal{F}$, and $e \in_{r}$ Key $\mathcal{F}$.

PROOF. Let us denote by $\mathcal{D}_{m}^{n}$ the distribution of the lower tuple; the upper tuple's distribution is thus $\mathcal{D}_{n}^{n}$.

From Lemma 1 , for all $j=m+1 \ldots n$, the distributions $\mathcal{D}_{j}^{n}$ and $\mathcal{D}_{j-1}^{n}$ are indistinguishable. (The first $j$ columns of $\mathcal{D}_{j}^{n}$ are identical to $\mathcal{D}_{j}$ of Lemma 1 , the first $j$ columns of $\mathcal{D}_{j-1}^{n}$ are identical to $\mathcal{D}_{j-1}$ of Lemma 1 , and the last $n-j$ columns of $\mathcal{D}_{j-1}^{n}$ and $\mathcal{D}_{j}^{n}$ are just uniformly random numbers.)
Since $\mathcal{D}_{j-1}^{n}$ and $\mathcal{D}_{j}^{n}$ are indistinguishable for $\forall j=m+1 \ldots n$, and because $n$ is bounded by a polynomial, $\mathcal{D}_{n}^{n}$ is also indistinguishable from any $\mathcal{D}_{m}^{n}$ (where $0 \leqslant m \leqslant n$ ). Let $\mathcal{A}_{k}$ be an algorithm that pretends to distinguish $\mathcal{D}_{n}^{n}$ from $\mathcal{D}_{m}^{n}$, and returns true or false. Now

$$
\begin{aligned}
& \operatorname{Pr}\left[\mathcal{A}_{k}(T) \mid T \sim \mathcal{D}_{n}^{n}\right]-\operatorname{Pr}\left[\mathcal{A}_{k}(T) \mid T \sim \mathcal{D}_{m}^{n}\right] \\
& =\sum_{j=m+1}^{n}\left(\operatorname{Pr}\left[\mathcal{A}_{k}(T) \mid T \sim \mathcal{D}_{j}^{n}\right]-\operatorname{Pr}\left[\mathcal{A}_{k}(T) \mid T \sim \mathcal{D}_{j-1}^{n}\right]\right)
\end{aligned}
$$

Here $k$ is the number of bits in the tuple values. Consider any polynomial $p(k)$; we want to prove that $\exists k_{0} \forall k \geqslant k_{0}$ the difference (1) is bounded by $1 / p(k)$. Let $p^{\prime}(k)=n p(k)$, which is also a polynomial. We have $\forall j=m+1 \ldots n \exists k_{j} \forall k \geqslant k_{j}$ the $j$-th difference in the telescoping sum is bounded by $1 / p^{\prime}(k)$. Now set $k_{0}=\max _{j} k_{j}$, and we are done:

$$
\begin{aligned}
\sum_{j=m+1}^{n}\left(\operatorname { P r } \left[\mathcal{A}_{k}(T) \mid T\right.\right. & \left.\left.\sim \mathcal{D}_{j}^{n}\right]-\operatorname{Pr}\left[\mathcal{A}_{k}(T) \mid T \sim \mathcal{D}_{j-1}^{n}\right]\right) \\
& <\sum_{j=m+1}^{n} \frac{1}{p^{\prime}(k)}<\frac{n}{n p(k)}=\frac{1}{p(k)}
\end{aligned}
$$

Therefore $\mathcal{D}_{n}^{n}$ and $\mathcal{D}_{m}^{n}$ are computationally indistinguishable.

STATEMENT 2. The intersection protocol is secure if both parties are semi-honest. In the end, $S$ learns only the size $\left|V_{R}\right|$, and $R$ learns only the size $\left|V_{S}\right|$ and the intersection $V_{S} \cap V_{R}$.

PROOF. We use a standard proof methodology from multi-party secure computation [26]. If, for any $V_{S}$ and $V_{R}$, the distribution of the $S$ 's view of the protocol (the information $S$ gets from $R$ ) cannot be distinguished from a simulation of this view that uses only $V_{S}$ and $\left|V_{R}\right|$, then clearly $S$ cannot learn anything from the inputs it gets from $R$ except for $\left|V_{R}\right|$. Note that the simulation only uses the knowledge $S$ is supposed to have at the end of the protocol, while the distinguisher also uses the inputs of $R$ (i.e., $V_{R}$ ), but not $R$ 's secret keys (i.e., $e_{R}$ ). It is important that the distinguisher be unable to distinguish between the simulation and the real view even given $R$ 's inputs: this precludes the kind of attack that broke the protocol given in Section 3.1.

The simulator for $S$ (that simulates what $S$ receives from $R$ ) is easy to construct. At Step 3 of the protocol, the only step where $S$ receives anything, the simulator generates $\left|V_{R}\right|$ random values $z_{i} \in_{r} \operatorname{Dom} \mathcal{F}$ and orders them lexicographically. In the real protocol, these values equal $f_{e_{R}}(h(v))$ for $v \in V_{R}$. Assuming that, for all $v \in V_{R}$, the hashes $h(v)$ are distributed uniformly at random (random oracle model), by Lemma 2 the distributions

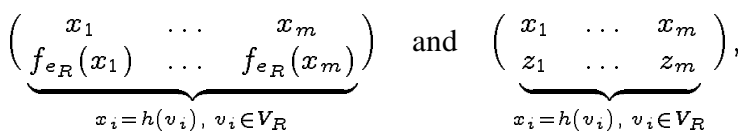

where $\forall i: z_{i} \in_{r} \operatorname{Dom} \mathcal{F}$, are indistinguishable. Therefore the real and simulated views for $S$ are also indistinguishable.

The simulator for $R$ (that simulates what $R$ gets from $S$ ) will use $V_{R}, V_{S} \cap V_{R}$ and $\left|V_{S}\right|$; it also knows the hash function $h$. However, it does not have $V_{S}-V_{R}$. The simulator chooses a key $\tilde{e}_{S} \in_{r}$ Key $\mathcal{F}$. In Step 4(a), the simulation creates $Y_{S}$ as follows:

- First, for values $v_{i} \in V_{S} \cap V_{R}$, the simulation adds $f_{\tilde{e}_{S}}\left(h\left(v_{i}\right)\right)$ to $Y_{S}$.

- Next, the simulation adds $\left|V_{S}-V_{R}\right|$ random values $z_{i} \epsilon_{r}$ $\operatorname{Dom} \mathcal{F}$ to $Y_{S}$. 
In Step 4(b), the simulation uses the key $\tilde{e}_{S}$ to encrypt each $y \in Y_{R}$. Since $e_{S}$ (real view) and $\tilde{e}_{S}$ (simulation) are both chosen at random, their distributions are identical. According to Lemma 2, one cannot distinguish between the distribution of

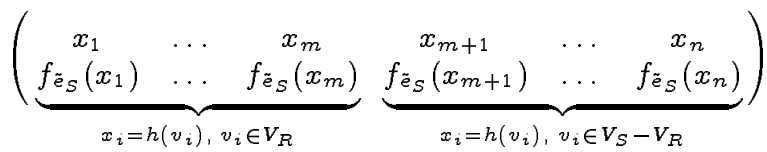

and the distribution of

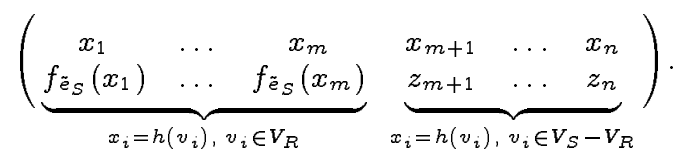

The real view corresponds to the upper matrix, and the simulated view to the lower matrix. The only difference is that some variables appear in the view encrypted by $f_{e_{R}}$, which makes the view a efficiently-computable function of the matrix. Therefore the real view and the simulated view are also indistinguishable, and the statement is proven.

\section{EQUIJOIN}

We now extend the intersection protocol so that, in addition to $V_{S} \cap V_{R}, R$ learns some extra information $\operatorname{ext}(v)$ from $S$ for values $v \in V_{S} \cap V_{R}$, but does not learn $\operatorname{ext}(v)$ for for $v \in V_{S}-V_{R}$. To compute the join $T_{S} \bowtie T_{R}$ on attribute $A$, we have $\operatorname{ext}(v)$ contain all the records of $S$ 's table where $T_{S} . A=v$, i.e., $\operatorname{ext}(v)$ contains the information about the other attributes in $T_{S}$ needed for the join.

\subsection{Idea Behind Protocol}

A simple, but incorrect, solution would be to encrypt the extra information $\operatorname{ext}(v)$ using $h(v)$ as the encryption key. Since, in our intersection protocol, $h(v)$ could not be discovered by $R$ except for $v \in V_{R}$ (and similarly for $S$ ), one might think that this protocol would be secure. While it is true that $h(v)$ cannot be discovered from $Y_{R}$ or $Y_{S}, h(v)$ can be discovered from the encryption of $\operatorname{ext}(v)$. For any arbitrary value $v, R$ can compute $h(v)$ and try decrypting all the $\operatorname{ext}(v)$ using $h(v)$ to learn whether or not $v \in V_{S}$. In fact, if the domain is small, $R$ can exhaustively go over all possible values and completely learn both $V_{S}$ and ext $(v)$ for $v \in V_{S}$.

Rather then encrypt the extra information with $h(v)$, we will encrypt it with a key $\kappa(v)=f_{e_{S}^{\prime}}(h(v))$, where $e_{S}^{\prime}$ is a second secret key of $S$. The problem now is to allow $R$ to learn $\kappa(v)$ for $v \in V_{R}$ without revealing $V_{R}$ to $S$. We do this as follows: $R$ sends $f_{e_{R}}(h(v))$ to $S$, and $S$ sends back $f_{e_{S}^{\prime}}\left(f_{e_{R}}(h(v))\right)$ to $R$. $R$ can now apply $f_{e_{R}}^{-1}$ to the latter to get

$$
f_{e_{R}}^{-1}\left(f_{e_{S}^{\prime}}\left(f_{e_{R}}(h(v))\right)\right)=f_{e_{R}}^{-1}\left(f_{e_{R}}\left(f_{e_{S}^{\prime}}(h(v))\right)\right)=f_{e_{S}^{\prime}}(h(v)) .
$$

Note that $R$ only gets $f_{e_{S}^{\prime}}(h(v))$ for $v \in V_{R}$, not for $v \in V_{S}-V_{R}$.

\subsection{Encryption Function $K$}

We now formally define the encryption function $K(\kappa, \operatorname{ext}(v))$ that encrypts $\operatorname{ext}(v)$ using the key $\kappa(v) . K$ is defined to be a function

$$
K: \operatorname{Dom} \mathcal{F} \times V_{\text {ext }} \rightarrow C_{\text {ext }}
$$

with two properties:

1. Each function $K_{\kappa}(x) \equiv K(\kappa, x)$ can be efficiently inverted (decrypted) given $\kappa$;

2. "Perfect Secrecy" [43]: For any $\operatorname{ext}(v)$, the value of $K_{\kappa}(\operatorname{ext}(v))$ is indistinguishable from a fixed (independent of $\operatorname{ext}(v)$ ) distribution $\mathcal{D}_{\text {ext }}$ over $C_{\text {ext }}$ when $\kappa \epsilon_{r} \operatorname{Dom} \mathcal{F}$.
Example 2 Let $\mathcal{F}$ be the power function over quadratic residues modulo a safe prime, as in Example 1. If the extra information $\operatorname{ext}(v)$ can also be encoded as a quadratic residue (i.e., $V_{\text {ext }}=$ Dom $\mathcal{F})$, the encryption $K_{\kappa}(\operatorname{ext}(v))$ can be just a multiplication operation:

$$
K_{\kappa}(\operatorname{ext}(v))=\kappa \cdot \operatorname{ext}(v) .
$$

The multiplication can be easily reversed given $\kappa$, and if $\kappa$ is uniformly random then $\kappa \cdot \operatorname{ext}(v)$ is also uniformly random (independently of ext $(v)$ ).

\subsection{Equijoin Protocol}

Let $V_{S}$ be the set of values (without duplicates) that occur in $T_{S} . A$, and let $V_{R}$ be the set of values that occur in $T_{R}$. $A$. For each $v \in V_{S}$, let $\operatorname{ext}(v)$ be all records in $T_{S}$ where $T_{S} . A=v$.

1. Both $S$ and $R$ apply hash function $h$ to their sets: $X_{S}=h\left(V_{S}\right)$ and $X_{R}=h\left(V_{R}\right)$.

$R$ chooses its secret key $e_{R} \in_{r}$ Key $\mathcal{F}$, and $S$ chooses two secret keys: $e_{S}, e_{S}^{\prime} \in_{r}$ Key $\mathcal{F}$.

2. $R$ encrypts its hashed set: $Y_{R}=f_{e_{R}}\left(X_{R}\right)=f_{e_{R}}\left(h\left(V_{R}\right)\right)$.

3. $R$ sends to $S$ its encrypted set $Y_{R}$, reordered lexicographically.

4. $S$ encrypts each $y \in Y_{R}$ with both key $e_{S}$ and key $e_{S}^{\prime}$, and sends back to $R$ 3-tuples $\left\langle y, f_{e_{S}}(y), f_{e_{S}^{\prime}}(y)\right\rangle$ $=\left\langle f_{e_{R}}(h(v)), f_{e_{S}}\left(f_{e_{R}}(h(v))\right), f_{e_{S}^{\prime}}\left(f_{e_{R}}(h(v))\right)\right\rangle$.

5. For each $v \in V_{S}, S$ does the following:

(a) Encrypts the hash $h(v)$ with $e_{S}$, obtaining $f_{e_{S}}(h(v))$.

(b) Generates the key for extra information using $e_{S}^{\prime}$ : $\kappa(v)=f_{e_{S}^{\prime}}(h(v))$.

(c) Encrypts the extra information: $c(v)=K(\kappa(v), \operatorname{ext}(v))$

(d) Forms a pair $\left\langle f_{e_{S}}(h(v)), c(v)\right\rangle$ $=\left\langle f_{e_{S}}(h(v)), K\left(f_{e_{S}^{\prime}}(h(v)), \operatorname{ext}(v)\right)\right\rangle$.

The pairs are then shipped to $R$ in lexicographical order.

6. $R$ applies $f_{e_{R}}^{-1}$ to all entries in the 3-tuples received at Step 4, obtaining $\left\langle h(v), f_{e_{S}}(h(v)), f_{e_{S}^{\prime}}(h(v))\right\rangle$ for all $v \in V_{R}$.

7. $R$ sets aside all pairs $\left\langle f_{e_{S}}(h(v)), K\left(f_{e_{s}^{\prime}}(h(v))\right.\right.$, ext $\left.\left.(v)\right)\right\rangle$ received at Step 5 whose first entry occurs as a second entry in a 3-tuple $\left\langle h(v), f_{e_{S}}(h(v)), f_{e_{S}^{\prime}}(h(v))\right\rangle$ from Step 6. Using the third entry $f_{e_{s}^{\prime}}(h(v))=\kappa(v)$ as the key, $R$ decrypts $K\left(f_{e_{S}^{\prime}}(h(v)), \operatorname{ext}(v)\right)$ and gets $\operatorname{ext}(v)$. The corresponding $v$ 's form the intersection $V_{S} \cap V_{R}$.

8. $R$ uses $\operatorname{ext}(v)$ for $v \in V_{S} \cap V_{R}$ to compute $T_{S} \bowtie T_{R}$.

\subsection{Proofs of Correctness and Security}

STATEMENT 3. Assuming there are no hash collisions, $S$ learns $\left|V_{R}\right|$, and $R$ learns $\left|V_{S}\right|, V_{S} \cap V_{R}$, and $\operatorname{ext}(v)$ for all $v \in V_{S} \cap V_{R}$.

PROOF. This protocol is an extension of the intersection protocol, so it allows $R$ to determine $V_{S} \cap V_{R}$ correctly. Since $R$ learns the keys $\kappa(v)$ for values in the intersection, $R$ also gets $\operatorname{ext}(v)$ for $v \in V_{S} \cap V_{R}$.

Next we prove that $R$ and $S$ do not learn anything besides the above. We first extend Lemma 2 as follows. 
LEMMA 3. For polynomial n, the distributions of the following two $3 \times n$-tuples

$$
\left(\begin{array}{ccc}
x_{1} & \ldots & x_{n} \\
f_{e}\left(x_{1}\right) & \ldots & f_{e}\left(x_{n}\right) \\
f_{e^{\prime}}\left(x_{1}\right) & \ldots & f_{e^{\prime}}\left(x_{n}\right)
\end{array}\right) \text { and }\left(\begin{array}{ccc}
x_{1} & \ldots & x_{n} \\
y_{1} & \ldots & y_{n} \\
z_{1} & \ldots & z_{n}
\end{array}\right)
$$

are computationally indistinguishable, where $\forall i: x_{i}, y_{i}, z_{i} \in_{r}$ $\operatorname{Dom} \mathcal{F}$, and $e, e^{\prime} \in_{r}$ Key $\mathcal{F}$.

Proof. Let us denote the left distribution by $\mathcal{D}_{1}$, the right distribution by $\mathcal{D}_{2}$, and the following "intermediate" distribution by $\mathcal{D}_{3}$ :

$$
\left(\begin{array}{ccc}
x_{1} & \cdots & x_{n} \\
f_{e}\left(x_{1}\right) & \cdots & f_{e}\left(x_{n}\right) \\
z_{1} & \cdots & z_{n}
\end{array}\right)
$$

The first and third line in the tuples for $\mathcal{D}_{1}$ and $\mathcal{D}_{3}$ are distributed like $\mathcal{D}_{n}^{n}$ and $\mathcal{D}_{0}^{n}$ (from Lemma 2) respectively. The second line in both $\mathcal{D}_{1}$ and $\mathcal{D}_{3}$ can be obtained from the first line by applying $f_{e}$ with random key $e$. Therefore, since $\mathcal{D}_{n}^{n}$ and $\mathcal{D}_{0}^{n}$ are indistinguishable by Lemma 2 , distributions $\mathcal{D}_{1}$ and $\mathcal{D}_{3}$ are also indistinguishable.

Analogously, the first and second lines in $\mathcal{D}_{3}$ and $\mathcal{D}_{2}$ are distributed like $\mathcal{D}_{0}^{n}$ and $\mathcal{D}_{n}^{n}$ respectively. The third line in both $\mathcal{D}_{3}$ and $\mathcal{D}_{2}$ can be obtained by using random numbers for the $z_{i}$ 's. Therefore, by Lemma $2, \mathcal{D}_{3}$ and $\mathcal{D}_{2}$ are also indistinguishable.

Finally, since both $\mathcal{D}_{1}$ and $\mathcal{D}_{2}$ are indistinguishable from $\mathcal{D}_{3}$, they themselves are indistinguishable.

The following lemma will be used in the proof for the security of the join protocol to show that the real and simulated views for $R$ are indistinguishable. $\mathcal{D}_{1}^{\prime}$ corresponds to the real view (for $R$ ), while $\mathcal{D}_{2}^{\prime}$ corresponds to the simulated view. The first $t$ columns correspond to $V_{S}-\left(V_{S} \cap V_{R}\right)$, the next $m-t$ columns to $V_{S} \cap V_{R}$, and the last $n-m$ columns to $V_{R}-\left(V_{S} \cap V_{R}\right)$.

LEMMA 4. For polynomial $m$, $t$, and $n$, and any $c_{i} \in V_{\text {ext }}$, the two distributions $\mathcal{D}_{1}^{\prime}$ and $\mathcal{D}_{2}^{\prime}$ of the $4 \times n$-tuple

$$
\left(\begin{array}{lllllllll}
x_{1} & \ldots & x_{t} & x_{t+1} & \ldots & x_{m} & x_{m+1} & \ldots & x_{n} \\
y_{1} & \ldots & y_{t} & y_{t+1} & \ldots & y_{m} & y_{m+1} & \ldots & y_{n} \\
& & & z_{t+1} & \ldots & z_{m} & z_{m+1} & \ldots & z_{n} \\
\xi_{1} & \ldots & \xi_{t} & \xi_{t+1} & \ldots & \xi_{m} & & &
\end{array}\right)
$$

such that

- For $\mathcal{D}_{1}^{\prime}, \forall i: x_{i} \in_{r} \operatorname{Dom} \mathcal{F}, y_{i}=f_{e}\left(x_{i}\right), z_{i}=f_{e^{\prime}}\left(x_{i}\right)$, and $\xi_{i}=K\left(f_{e^{\prime}}\left(x_{i}\right), c_{i}\right)$ where $e, e^{\prime} \in_{r} \operatorname{Key} \mathcal{F}$;

- For $\mathcal{D}_{2}^{\prime}, \forall i: x_{i}, y_{i}, z_{i} \in_{r} \operatorname{Dom} \mathcal{F}$, and

$-i=1 \ldots t: \xi_{i}$ is independent random with distribution $\mathcal{D}_{\text {ext }}$,

- $i=t+1 \ldots m: \xi_{i}=K\left(z_{i}, c_{i}\right)$

are computationally indistinguishable. (In both $\mathcal{D}_{1}^{\prime}$ and $\mathcal{D}_{2}^{\prime}$, the positions corresponding to $z_{1} \ldots z_{t}$ and $\xi_{m+1} \ldots \xi_{n}$ are blank.) tion:

Proof. Denote by $\mathcal{D}_{3}^{\prime}$ the following "intermediate" distribu-

$$
\forall i: x_{i}, y_{i}, z_{i} \in_{r} \operatorname{Dom} \mathcal{F} \text { and } \xi_{i}=K\left(z_{i}, c_{i}\right) .
$$

Note that the $z_{i}$ for $i=1 \ldots t$ are not included in the tuple, even though they are used to generate $K\left(z_{i}, c_{i}\right)$.

The only difference between the two distributions $\mathcal{D}_{2}^{\prime}$ and $\mathcal{D}_{3}^{\prime}$ is that, for $i=1 \ldots t$, we replace $\xi_{i}$ distributed as $\mathcal{D}_{\text {ext }}$ with $K\left(z_{i}, c_{i}\right)$ where $z_{i} \in_{r} \operatorname{Dom} \mathcal{F}$; the rest of the matrix is independent and stays the same. Since $z_{i}$ is not a part of the matrix for $i=1 \ldots t$, by Property 2 of encryption $K(\kappa, c)$, distributions $\mathcal{D}_{2}^{\prime}$ and $\mathcal{D}_{3}^{\prime}$ are indistinguishable.

Next we use Lemma 3 to show that distributions $\mathcal{D}_{1}^{\prime}$ and $\mathcal{D}_{3}^{\prime}$ are also indistinguishable. We define function $Q(M)$ that takes a $3 \times n$ matrix $M$ (from Lemma 3) and generates a $4 \times n$ matrix $M^{\prime}$ as follows:

1. The first 3 rows of $M^{\prime}$ are the same as the first 3 rows of $M$, except that the values corresponding to $z_{1}, \ldots, z_{t}$ in $M^{\prime}$ are left blank.

2. The fourth row of $M^{\prime}$ is generated by taking $\xi_{i}=K\left(z_{i}, c_{i}\right)$ where $z_{i}$ is the corresponding value of the third row of $M$.

If $M$ is distributed like $\mathcal{D}_{1}$ of Lemma $3, Q(M)$ corresponds to $\mathcal{D}_{1}^{\prime}$. If $M$ is distributed like $\mathcal{D}_{2}, Q(M)$ corresponds to $\mathcal{D}_{3}^{\prime}$. Since by Lemma $3, \mathcal{D}_{1}$ and $\mathcal{D}_{2}$ are indistinguishable, and $Q(M)$ is computable in polynomial time, $\mathcal{D}_{1}^{\prime}$ and $\mathcal{D}_{3}^{\prime}$ are also indistinguishable.

Finally, since both $\mathcal{D}_{1}^{\prime}$ and $\mathcal{D}_{2}^{\prime}$ are indistinguishable from $\mathcal{D}_{3}^{\prime}$, they themselves are indistinguishable.

STATEMENT 4. The join protocol is secure if both parties are semi-honest. At the end of the protocol, $S$ learns only $\left|V_{R}\right| ; R$ learns only $\left|V_{S}\right|, V_{S} \cap V_{R}$, and $\operatorname{ext}(v)$ for all $v \in V_{S} \cap V_{R}$.

PROOF. As in the proof of Statement 2, we will construct simulators of each party's view of the protocol, such that each simulator is given only what the party is supposed to learn, and such that the distribution of the real view is indistinguishable from the distribution of the simulated view.

The simulator for $S$ is identical to that in Statement 2, since $S$ gets exactly the same input from $R$ as in the intersection protocol. Hence the proof from Statement 2 directly applies.

The simulator for $R$ (that simulates what $R$ receives from $S$ ) can use $h, e_{R}, V_{R}, V_{S} \cap V_{R}$, ext $(v)$ for $v \in V_{S} \cap V_{R}$, and $\left|V_{S}\right|$. Let

$$
\begin{aligned}
V_{S} & =\left\{v_{1}, \ldots, v_{t}, v_{t+1}, \ldots, v_{m}\right\} \text { and } \\
V_{R} & =\left\{v_{t+1}, \ldots, v_{m}, v_{m+1}, \ldots, v_{n}\right\} .
\end{aligned}
$$

So $t=\left|V_{S}-V_{R}\right|, m=\left|V_{S}\right|$, and $n=\left|V_{S} \cup V_{R}\right|$. Note that the simulator does not know the values in $V_{S}-V_{R}$.

In Step 4, the simulator generates $n$ random numbers $y_{i} \in_{r}$ Dom $\mathcal{F}, i=1 \ldots n$ as the simulated values for $f_{e_{S}}\left(h\left(v_{i}\right)\right)$, and an additional $n$ random numbers $z_{i} \in_{r}$ Dom $\mathcal{F}$ as the simulated values for $f_{e_{S}^{\prime}}\left(h\left(v_{i}\right)\right)$. The simulation then uses key $e_{R}$ to create

$$
\left\langle f_{e_{R}}\left(h\left(v_{i}\right)\right), f_{e_{R}}\left(y_{i}\right), f_{e_{R}}\left(z_{i}\right)\right\rangle
$$

for $i=t+1 \ldots m$. These triplets are ordered lexicographically and comprise the simulated view for Step 4.

In Step 5, the simulator creates the pairs as follows:

- For values $v_{t+1}, \ldots, v_{m}$ from $V_{S} \cap V_{R}$, the simulator encrypts $\operatorname{ext}\left(v_{i}\right)$ as $\xi_{i}=K\left(z_{i}, \operatorname{ext}\left(v_{i}\right)\right)$; then it forms pairs $\left\langle y_{i}, \xi_{i}\right\rangle$;

- For $i=1 \ldots t$, the simulator creates $\left|V_{S}-V_{R}\right|$ additional pairs $\left\langle y_{i}, \xi_{i}\right\rangle$ where $\xi_{i}$ have distribution $\mathcal{D}_{\text {ext }}$ over $C_{\text {ext }}$, i.e., $y_{i}$ and $\xi_{i}$ are random values from their respective domains.

These pairs are sorted lexicographically and comprise the simulated view for Step 5.

Setting $x_{i}=h\left(v_{i}\right)$, the real view corresponds to distribution $\mathcal{D}_{1}^{\prime}$ of the matrix in Lemma 4, while the simulation corresponds to distribution $\mathcal{D}_{2}^{\prime}$ of the matrix. The only difference is that some variables appear in the view encrypted by $f_{e_{R}}$, which makes the view a efficiently-computable function of the matrix. Since these $\mathcal{D}_{1}^{\prime}$ and $\mathcal{D}_{2}^{\prime}$ are indistinguishable, the simulation is also indistinguishable from the real view. 


\section{INTERSECTION AND JOIN SIZES}

\subsection{Intersection Size}

We now show how the intersection protocol can be modified, such that $R$ only learns the intersection size, but not which values in $V_{R}$ were present in $V_{S}$. (Simply applying the intersection protocol would reveal the set $V_{R} \cap V_{S}$, in addition to the intersection size.) Recall that in Step 4 of the intersection protocol, $S$ sends back to $R$ the values of $y \in Y_{R}$ together with their encryptions made by $S$. These encryptions are paired with the unencrypted $y$ 's so that $R$ can match the encryptions with $R$ 's values. If instead $S$ sends back to $R$ only the lexicographically reordered encryptions of the $y$ 's and not the $y$ 's themselves, $R$ can no longer do the matching.

\subsubsection{Intersection Size Protocol}

We now present the protocol for intersection size. (Steps 1 through 3 are the same as in the intersection protocol.)

1. Both $S$ and $R$ apply hash function $h$ to their sets: $X_{S}=h\left(V_{S}\right)$ and $X_{R}=h\left(V_{R}\right)$.

Each party randomly chooses a secret key: $e_{S} \in_{r}$ Key $\mathcal{F}$ for $S$ and $e_{R} \in_{r}$ Key $\mathcal{F}$ for $R$.

2. Both parties encrypt their hashed sets:

$Y_{S}=f_{e_{S}}\left(X_{S}\right)=f_{e_{S}}\left(h\left(V_{S}\right)\right)$ and $Y_{R}=f_{e_{R}}\left(X_{R}\right)=f_{e_{R}}\left(h\left(V_{R}\right)\right)$.

3. $R$ sends to $S$ its encrypted set $Y_{R}=f_{e_{R}}\left(h\left(V_{R}\right)\right)$, reordered lexicographically.

4. (a) $S$ ships to $R$ its set $Y_{S}=f_{e_{S}}\left(h\left(V_{S}\right)\right)$, reordered lexicographically.

(b) $S$ encrypts each $y \in Y_{R}$ with $S$ 's key $e_{S}$ and sends back to $R$ the set $Z_{R}=f_{e_{S}}\left(Y_{R}\right)=f_{e_{S}}\left(f_{e_{R}}\left(h\left(V_{R}\right)\right)\right)$, reordered lexicographically.

5. $R$ encrypts each $y \in Y_{S}$ with $e_{R}$, obtaining $Z_{S}=f_{e_{R}}\left(f_{e_{S}}\left(h\left(V_{S}\right)\right)\right)$.

6. Finally, $R$ computes intersection size $\left|Z_{S} \cap Z_{R}\right|$, which equals $\left|V_{S} \cap V_{R}\right|$.

\subsubsection{Proofs of Correctness and Security}

STATEMENT 5. Assuming there are no hash collisions, $S$ learns the size $\left|V_{R}\right|$ and $R$ learns the size $\left|V_{S}\right|$ and the size $\left|V_{S} \cap V_{R}\right|$.

PROOF. The proof is very similar to that for Statement 1 . Since $f_{e_{S}}$ and $f_{e_{R}}$ commute and are bijective, assuming that hash function $h$ has no collisions on $V_{S} \cup V_{R}$,

$$
\left|V_{S} \cap V_{R}\right|=f_{e_{R}}\left(f_{e_{S}}\left(h\left(V_{S}\right)\right)\right) \cap f_{e_{S}}\left(f_{e_{R}}\left(h\left(V_{R}\right)\right)\right) .
$$

Therefore $R$ recovers the correct size $\left|V_{S} \cap V_{R}\right|$.

STATEMENT 6. The intersection size protocol is secure if both parties are semi-honest. At the end of the protocol, $S$ learns only the size $\left|V_{R}\right|$, and $R$ learns only the sizes $\left|V_{S}\right|$ and $\left|V_{S} \cap V_{R}\right|$.

PROOF. We use the same methodology as in the proofs of Statement 2 and 4.

The simulator for $S$ 's view of the intersection size protocol is identical to that in Statement 2, since $S$ gets exactly the same input from $R$ as in the intersection protocol. Hence the proof from Statement 2 directly applies.
The simulator for $R$ 's view of the protocol is allowed to use $V_{R}$, the hash function $h, e_{R}$, and the numbers $\left|V_{S} \cap V_{R}\right|$ and $\left|V_{S}\right|$; however, it has neither $V_{S}-V_{R}$ nor $V_{S} \cap V_{R}$. Let

$$
\begin{aligned}
& V_{S}=\left\{v_{1}, \ldots, v_{t}, v_{t+1}, \ldots, v_{m}\right\} \text { and } \\
& V_{R}=\left\{v_{t+1}, \ldots, v_{m}, v_{m+1}, \ldots, v_{n}\right\} .
\end{aligned}
$$

So $t=\left|V_{S}-V_{R}\right|, m=\left|V_{S}\right|$, and $n=\left|V_{S} \cup V_{R}\right|$.

The simulator generates $n$ random numbers $y_{1}, \ldots, y_{n} \in_{r}$ Dom $\mathcal{F}$ which play the role of $f_{e_{S}}(h(v))$ for all $v \in V_{S} \cup V_{R}$. The key $e_{S}$ is not simulated, and no decision is made about which $y_{i}$ stands for which $f_{e_{S}}(h(v))$. In Step 4(a), the simulation creates $Y_{S}$ as

$$
Y_{S}=\left\{y_{1}, \ldots, y_{m}\right\} .
$$

In Step 4(b), the simulation generates $Z_{R}$ by taking set $\left\{y_{t+1}, \ldots, y_{n}\right\}$ and encoding it with $f_{e_{R}}$ :

$$
Z_{R}=\left\{f_{e_{R}}\left(y_{t+1}\right), \ldots, f_{e_{R}}\left(y_{n}\right)\right\} .
$$

We now show that the distribution of $R$ 's real view in the protocol is computationally indistinguishable from the distribution of $R$ 's simulated view.

According to Lemma 2, the distributions $\mathcal{D}_{0}^{n}$ and $\mathcal{D}_{n}^{n}$ of the following matrix $M$ :

$$
\left(\begin{array}{lll}
x_{1} & \ldots & x_{n} \\
y_{1} & \ldots & y_{n}
\end{array}\right)
$$

where

- $\mathcal{D}_{0}^{n}: \forall i: x_{i}, y_{i} \in_{r} \operatorname{Dom} \mathcal{F}$;

- $\mathcal{D}_{n}^{n}: \forall i: x_{i} \in_{r} \operatorname{Dom} \mathcal{F}, y_{i}=f_{e_{S}}\left(x_{i}\right), e_{S} \in_{r} \operatorname{Key} \mathcal{F}$;

are indistinguishable. Given $x_{i}=h\left(v_{i}\right)$, consider the following function $Q(M)$ :

$$
Q(M)=\left\langle h, e_{R}, Y_{S}, Z_{R}\right\rangle
$$

where

$$
\begin{aligned}
h & :=\text { a function on } V_{S} \cup V_{R} \text { s.t. } \forall i: h\left(v_{i}\right)=x_{i} ; \\
e_{R} & :=\text { a random key; } \\
Y_{S} & :=\left\{y_{1}, \ldots, y_{m}\right\} ; \\
Z_{R} & :=\left\{f_{e_{R}}\left(y_{t+1}\right), \ldots, f_{e_{R}}\left(y_{n}\right)\right\} .
\end{aligned}
$$

If $M$ is distributed according to $\mathcal{D}_{0}^{n}$, then $Q(M)$ corresponds to the simulated view of server $R$. If $M$ 's distribution is $\mathcal{D}_{n}^{n}$, then

$$
\begin{aligned}
& y_{i}=f_{e_{S}}\left(x_{i}\right)=f_{e_{S}}\left(h\left(v_{i}\right)\right), \\
& f_{e_{R}}\left(y_{i}\right)=f_{e_{R}}\left(f_{e_{S}}\left(x_{i}\right)\right)=f_{e_{S}}\left(f_{e_{R}}\left(h\left(v_{i}\right)\right)\right),
\end{aligned}
$$

and $Q(M)$ is distributed like the real view of $R$. Since from Lemma $2, \mathcal{D}_{0}^{n}$ and $\mathcal{D}_{n}^{n}$ are indistinguishable, and $Q$ is computable in polynomial time, the simulated view $Q\left(\mathcal{D}_{0}^{n}\right)$ and the real view $Q\left(\mathcal{D}_{n}^{n}\right)$ are also indistinguishable.

\subsection{Equijoin Size}

To evaluate equijoin size, we follow the intersection size protocol, except that we allow $V_{R}$ and $V_{S}$ to be multi-sets, i.e., contain duplicates, and then compute the join size instead of the intersection size in Step 6. However, $R$ can now use the number of duplicates of a given value to partially match values in $Y_{R}$ with their corresponding encryptions in $Z_{R}$. We now characterize exactly what $R$ and $S$ learn in this protocol (besides $\left|V_{R}\right|,\left|V_{S}\right|$ and $\left|V_{R} \bowtie V_{S}\right|$ ).

To start with, $R$ learns the distribution of duplicates in $V_{S}$, and $S$ learns the distribution of duplicates in $V_{R}$. To characterize what else $R$ learns, let us partition the values in $V_{R}$ based on the number of duplicates, i.e., in a partition $V_{R}(d)$, each $v \in V_{R}(d)$ has 
$d$ duplicates. Then, for each partition, $R$ learns $\left|V_{R}(d) \cap V_{S}\left(d^{\prime}\right)\right|$ for each partition $V_{S}\left(d^{\prime}\right)$ of $V_{S}$. Thus if all values have the same number of duplicates (e.g., no duplicates as in our intersection protocol), $R$ only learns $\left|V_{R} \cap V_{S}\right|$. At the other extreme, if no two values have the same number of duplicates, $R$ will learn $V_{R} \cap V_{S}$.

\section{COST ANALYSIS}

\subsection{Protocols}

Let

- each encrypted codeword (in Dom $\mathcal{F}$ ) be $k$ bits long,

- $C_{h}$ denote the cost of evaluating the hash function,

- $C_{e}$ denote the cost of encryption/decryption by $\mathcal{F}$ (e.g., exponentiation " $x^{y} \bmod p$ " over $k$-bit integers),

- $C_{K}$ denote the cost of encryption/decryption by $K$ (e.g., encoding/decoding as a quadratic residue and multiplication), and

- $n \log n \cdot C_{s}$ be the cost of sorting a set of $n$ encryptions.

We assume the obvious optimizations when computing the computation and communication costs. For example, in the join protocol, we assume that the protocol does not decrypt $y$ to $h(v)$ in Step 6, but uses order preservation for matching. Also, in all the protocols, $S$ does not retransmit the $y$ 's back but just preserves the original order.

Computation The computation costs are:

- Intersection: $\left(C_{h}+2 C_{e}\right)\left(\left|V_{S}\right|+\left|V_{R}\right|\right)+2 C_{s}\left|V_{S}\right| \log \left|V_{S}\right|$ $+3 C_{s}\left|V_{R}\right| \log \left|V_{R}\right|$

- Join: $C_{h}\left(\left|V_{S}\right|+\left|V_{R}\right|\right)+2 C_{e}\left|V_{S}\right|+5 C_{e}\left|V_{R}\right|$ $+C_{K}\left(\left|V_{S}\right|+\left|V_{S} \cap V_{R}\right|\right)+2 C_{s}\left|V_{S}\right| \log \left|V_{S}\right|$ $+3 C_{s}\left|V_{R}\right| \log \left|V_{R}\right|$

We can assume $C_{e} \gg C_{h}, C_{e} \gg C_{K}$, and $n C_{e} \gg n \log n \cdot C_{s}$, so these formulae can be approximated by

- Intersection: $2 C_{e}\left(\left|V_{S}\right|+\left|V_{R}\right|\right)$

- Join: $2 C_{e}\left|V_{S}\right|+5 C_{e}\left|V_{R}\right|$

Communication The communication cost is:

- Intersection: $\left(\left|V_{S}\right|+2\left|V_{R}\right|\right) \cdot k$ bits

- Join: $\left(\left|V_{S}\right|+3\left|V_{R}\right|\right) \cdot k+\left|V_{S}\right| \cdot k^{\prime}$ bits, where $k^{\prime}$ is the size of the encrypted ext $(v)$.

Both the intersection size and join size protocols have the same computation and communication complexity as the intersection protocol.

\subsection{Applications}

We now estimate the execution times for the applications in Section 1.1 .

For the cost of $C_{e}$ (i.e., cost of $x^{y} \bmod p$ ), we use the times from [36]: 0.02 s for 1024-bit numbers on a Pentium III (in 2001). This corresponds to around $2 \times 10^{5}$ exponentiations per hour. We assume that communication is via a T1 line, with bandwidth of 1.544 Mbits/second ( $\approx 5$ Gbits/hour).

Encrypting the set of values is trivially parallelizable in all three protocols. We assume that we have $P$ processors that we can utilize in parallel: we will use a default value of $P=10$.

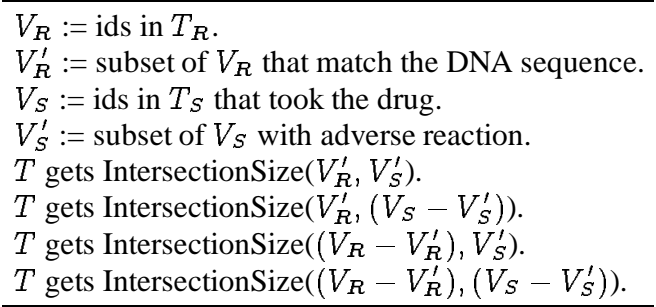

Figure 2: Algorithm for Medical Research Application

\subsubsection{Selective Document Sharing}

Recall that we have two databases $D_{R}$ and $D_{S}$, where each database contains a set of documents, and a document consists of a set of significant words. We wish to find all pairs of documents $d_{R} \in D_{R}$ and $d_{S} \in D_{S}$ such that, for some similarity function $f$ and threshold $\tau, f\left(\left|d_{R} \cap d_{S}\right|,\left|d_{R}\right|,\left|d_{S}\right|\right)>\tau$. For example, $f$ could be $\left|d_{R} \cap d_{S}\right| /\left(\left|d_{R}\right|+\left|d_{S}\right|\right)$.

Implementation $R$ and $S$ execute the intersection size protocol for each pair of documents $d_{R} \in D_{R}$ and $d_{S} \in D_{S}$ to get $\mid d_{R} \cap$ $d_{S}|,| d_{R} \mid$ and $\left|d_{S}\right|$; they then compute the similarity function $f$.

For $S$, in addition to the number of documents $\left|D_{S}\right|$, this protocol also reveals to $R$ for each document $d_{R} \in D_{R}$, which documents in $D_{S}$ matched $d_{R}$, and the size of $\left|d_{R} \cap d_{S}\right|$ for each document $d_{S} \in D_{S}$.

Cost Analysis For a given pair of documents $d_{R}$ and $d_{S}$, the computation time is $\left(\left|d_{R}\right|+\left|d_{S}\right|\right) \cdot 2 C_{e}$, and the data transferred is $\left(\left|d_{R}\right|+2\left|d_{S}\right|\right) \cdot k$ bits. Thus the total cost is:

- Computation: $\left|D_{R}\right| \cdot\left|D_{S}\right| \cdot\left(\left|d_{R}\right|+\left|d_{S}\right|\right) \cdot 2 C_{e}$.

- Communication: $\left|D_{R}\right| \cdot\left|D_{S}\right| \cdot\left(\left|d_{R}\right|+2\left|d_{S}\right|\right) \cdot k$.

If $\left|D_{R}\right|=10$ documents, $D_{S}=100$ documents, and $\left|d_{R}\right|=$ $\left|d_{S}\right|=1000$ words, the computation time will be $4 \times 10^{6} C_{e} / P \approx$ 2 hour. The data transferred will be $3 \times 10^{6} k \approx 3$ Gbits $\approx 35$ minutes.

\subsubsection{Medical Research}

Recall that we wish to get the answer to the query

$$
\begin{aligned}
& \text { select pattern, reaction, count(*) } \\
& \text { from } T_{R}, T_{S} \\
& \text { where } T_{R} \text {.id }=T_{S} \text {.id and } T_{S} \cdot \text { drug }=\text { true } \\
& \text { group by } T_{R} \text {.pattern, } T_{S} \text {.reaction }
\end{aligned}
$$

where $T_{R}$ and $T_{S}$ are tables in two different enterprises.

Implementation Figure 2 shows the implementation algorithm. We use a slightly modified version of the intersection size protocol where $Z_{R}$ and $Z_{S}$ are sent to $T$, the researcher, instead of to $S$ and $R$. Note that whenever we have, say, $\left(V_{R}-V_{R}^{\prime}\right)$ inside IntersectionSize, the set difference is computed locally, and the result is the input to the protocol.

Cost Analysis The combined cost of the four intersections is $2\left(\left|V_{R}\right|+\left|V_{S}\right|\right) \cdot 2 C_{e}$, and the data transferred is $2\left(\left|V_{R}\right|+\left|V_{S}\right|\right) \cdot 2 k$ bits. If $\left|V_{R}\right|=\left|V_{S}\right|=1$ million, the total computation time will be $8 \times 10^{6} C_{e} / P \approx 4$ hours. The total communication time will be $8 \times 10^{6} k \approx 8$ Gbits $\approx 1.5$ hours.

\section{CONCLUSIONS}

We identified information integration with minimal sharing as a new area for future database research. We developed novel protocols for three key operations: intersection, intersection size, and 
equijoin and proved that these protocols disclose minimal information apart from the query result. We also gave a protocol for computing equijoin size, but this protocol leaks some information about which tuples joined, based on the distribution of duplicates. We also showed how new applications can be built using the proposed protocols.

Some interesting directions for future research include:

- What is the tradeoff between the additional information being disclosed and efficiency? Will we be able to obtain much faster protocols if we are willing to disclose additional information?

- Can we formalize models of minimal disclosure and discover corresponding protocols for other database operations such as aggregations?

Acknowledgments We thank Robert Morris for suggesting motivating applications. We also thank Dalit Naor for pointing out related work in secure multi-party computation.

\section{REFERENCES}

[1] N. R. Adam and J. C. Wortman. Security-control methods for statistical databases. ACM Computing Surveys, 21(4):515556, Dec. 1989.

[2] R. Agrawal and J. Kiernan. Watermarking relational databases. In 28th Int'l Conference on Very Large Databases, Hong Kong, China, August 2002.

[3] R. Agrawal, J. Kiernan, R. Srikant, and Y. Xu. Hippocratic databases. In Proc. of the 28th Int'l Conference on Very Large Databases, Hong Kong, China, August 2002.

[4] R. Agrawal, J. Kiernan, R. Srikant, and Y. Xu. Implementing P3P using database technology. In Proc. of the 19th Int'l Conference on Data Engineering, Bangalore, India, March 2003.

[5] R. Agrawal, J. Kiernan, R. Srikant, and Y. Xu. An XPathbased preference language for P3P. In Proc. of the 12th Int'l World Wide Web Conference, Budapest, Hungary, May 2003.

[6] R. Agrawal and R. Srikant. Privacy preserving data mining. In ACM SIGMOD Conference on Management of Data, pages 439-450, Dallas, Texas, May 2000.

[7] S. Ajmani, R. Morris, and B. Liskov. A trusted third-party computation service. Technical Report MIT-LCS-TR-847, MIT, May 2001.

[8] M. Bellare and P. Rogaway. Random oracles are practical: A paradigm for designing efficient protocols. In Proc. of the First ACM Conf. on Computer and Communications Security, pages 62-73, Fairfax, Virginia, 1993.

[9] J. C. Benaloh and M. de Mare. One-way accumulators: A decentralized alternative to digital sinatures. In Advances in Cryptology-EUROCRYPT'93, Workshop on the Theory and Application of Cryptographic Techniques, volume 765 of Lecture Notes in Computer Science, pages 274-285, Lofthus, Norway, May 1993. Springer-Verlag.

[10] D. Boneh. The decision diffie-hellman problem. In Proc. of the 3rd International Algorithmic Number Theory Symposium, volume 1423 of Lecture Notes in Computer Science, pages 48-63, Portland, Oregon, USA, June 1998. SpringerVerlag.

[11] C. Cachin, S. Micali, and M. Stadler. Computationally private information retrieval with polylogarithmic communication. In Theory and Application of Cryptographic Techniques, pages 402-414, 1999.

[12] S. Chawathe, H. Garcia-Molina, J. Hammer, K. Ireland, Y. Papakonstantinou, J. Ullman, and J. Widom. The TSIMMIS project: Integration of heterogeneous information sources. In 16th Meeting of the Information Processing Society of Japan, pages 7-18, Tokyo, Japan, 1994.

[13] F. Chin and G. Ozsoyoglu. Auditing and infrence control in statistical databases. IEEE Transactions on Software Eng., SE-8(6):113-139, April 1982.

[14] B. Chor and N. Gilboa. Computationally private information retrieval. In Proc. of 29th ACM Symposium on Theory of Computing, pages 304-313, 1997.

[15] B. Chor, O. Goldreich, E. Kushilevitz, and M. Sudan. Private information retrieval. In IEEE Symposium on Foundations of Computer Science, pages 41-50, 1995.

[16] U. Dayal and H.-Y. Hwang. View definition and generalization for database integration in a multidatabase system. IEEE Transactions on Software Eng., 10(6):628-645, 1984.

[17] D. Denning, P. Denning, and M. Schwartz. The tracker: A threat to statistical database security. ACM Transactions on Database Systems, 4(1):76-96, March 1979.

[18] W. Diffie and M. Hellman. New directions in cryptography. IEEE Transactions on Information Theory, IT-22(6):644654, November 1976.

[19] D. Dobkin, A. Jones, and R. Lipton. Secure databases: Protection against user influence. ACM Transactions on Database Systems, 4(1):97-106, March 1979.

[20] T. ElGamal. A public key cryptosystem and a signature scheme based on discrete logarithms. IEEE Transactions on Information Theory, IT-31(4):469-472, July 1985.

[21] A. Elmagarmid, M. Rusinkiewicz, and A. Sheth, editors. Management of Heterogeneous \& Autonomous Database Systems. Morgan Kaufmann, 1999.

[22] A. Evfimievski, R. Srikant, R. Agrawal, and J. Gehrke. Privacy preserving mining of association rules. In Proc. of the 8th ACM SIGKDD Int'l Conference on Knowledge Discovery and Data Mining, Edmonton, Canada, July 2002.

[23] I. Fellegi. On the question of statistical confidentiality. Journal of the American Statistical Assoc., 67(337):7-18, March 1972.

[24] A. Fiat and A. Shamir. How to prove yourself: Practical solutions to identification and signature problems. In A. M. Odlyzko, editor, Advances in Cryptology, Proceedings of Crypto 86, volume 263 of Lecture Notes in Computer Science, pages 186-194. Springer-Verlag, 1987.

[25] Y. Gertner, Y. Ishai, E. Kushilevitz, and T. Malkin. Protecting data privacy in private information retrieval schemes. In $A C M$ Symposium on Theory of Computing, pages 151-160, 1998.

[26] O. Goldreich. Secure multi-party computation. Working Draft, Version 1.3, June 2001.

[27] L. M. Haas, R. J. Miller, B. Niswonger, M. T. Roth, P. M. Schwarz, and E. L. Wimmers. Transforming heterogeneous data with database middleware: Beyond integration. IEEE Data Engineering Bulletin, 22(1), 1999.

[28] B. A. Huberman, M. Franklin, and T. Hogg. Enhancing privacy and trust in electronic communities. In Proc. of the 1st ACM Conference on Electronic Commerce, pages 78-86, Denver, Colorado, November 1999.

[29] P. Ipeirotis and L. Gravano. Distributed search over the hidden web: Hierarchical database sampling and selection. In 28th Int'l Conference on Very Large Databases, Hong Kong, China, August 2002.

[30] N. Jefferies, C. Mitchell, and M. Walker. A proposed architecture for trusted third party services. In Cryptography Pol- 
icy and Algorithms Conference. Springer LNCS v 1029 pp 98-104, July 1995.

[31] M. Kantarcioglu and C. Clifton. Privacy-preserving distributed mining of association rules on horizontally partitioned data. In ACM SIGMOD Workshop on Research Issues on Data Mining and Knowledge Discovery, June 2002.

[32] E. Kushilevitz and R. Ostrovsky. Replication is not needed: Single database, computationally-private information retrieval. In Proc. of the IEEE Symp. on Found. of Computer Science (FOCS), 1997.

[33] Y. Lindell and B. Pinkas. Privacy preserving data mining. Journal of Cryptology, 15(3):177-206, 2002.

[34] M. Naor and K. Nissim. Communication preserving protocols for secure function evaluation. In Proc. of the ACM Symposium on Theory of Computing, pages 590-599, 2001.

[35] M. Naor and B. Pinkas. Oblivious transfer and polynomial evaluation. In Proc. of the 31th ACM Symposium on Theory of Computing, pages 245-254, Atlanta, Georgia, 1999.

[36] M. Naor and B. Pinkas. Efficient oblivious transfer protocols. In Proc. of the 12th Annual ACM-SIAM Symposium on Discrete Algorithms, pages 448-457, Washington DC, USA, January 2001.

[37] M. Naor, B. Pinkas, and R. Sumner. Privacy preserving auctions and mechanism design. In Proc. of the 1st ACM Conference on Electronic Commerce, pages 129-139, Denver, Colorado, November 1999.

[38] B. Preneel. Analysis and design of cryptographic hash functions. Ph.D. dissertation, Katholieke Universiteit Leuven, 1992.

[39] M. O. Rabin. How to exchange secrets by oblivious transfer. Technical Memo TR-81, Aiken Computation Laboratory, Harvard University, 1981.

[40] S. J. Rizvi and J. R. Haritsa. Privacy-preserving association rule mining. In Proc. of the 28th Int'l Conference on Very Large Databases, August 2002.

[41] G. Salton and M. McGill. Introduction to Modern Information Retrieval. McGraw-Hill, New York, 1983.

[42] A. Shamir, R. L. Rivest, and L. M. Adleman. Mental poker. Technical Memo MIT-LCS-TM-125, Laboratory for Computer Science, MIT, February 1979.

[43] C. E. Shannon. Communication theory of secrecy systems. Bell System Technical Journal, 28-4:656-715, 1949.

[44] A. Shoshani. Statistical databases: Characteristics, problems and some solutions. In Proc. of the Eighth Int'l Conference on Very Large Databases, pages 208-213, Mexico City, Mexico, September 1982.

[45] S. W. Smith and D. Safford. Practical private information retrieval with secure coprocessors. Research Report RC 21806, IBM, July 2000.

[46] D. R. Stinson. Cryptography: Theory and Practice, chapter 4. Chapman \& Hall/CRC, second edition, 2002.

[47] J. Vaidya and C. W. Clifton. Privacy preserving association rule mining in vertically partitioned data. In Proc. of the 8th ACM SIGKDD Int'l Conference on Knowledge Discoveryand Data Mining, Edmonton, Canada, July 2002.

[48] G. Wiederhold. Intelligent integration of information. In Proc. of the ACM SIGMOD Conf. on Management of Data, Washington, DC, 1993.

[49] A. C. Yao. How to generate and exchange secrets. In Proc. of the 27th Annual Symposium on Foundations of Computer Science, pages 162-167, Toronto, Canada, October 1986.

\section{APPENDIX}

\section{A. CIRCUIT-BASED PROTOCOLS}

For comparison, we estimate the computation and communication cost of intersection and join protocols obtained using the semihonest variant of Yao's protocol described in $[33,37]$. Let $V_{S}$ and $V_{R}$ contain $w$-bit values. Consider a function $f(\vec{x}, \vec{y})$ that takes vectors $\vec{x}$ and $\vec{y}$ (of size $w \cdot\left|V_{S}\right|$ and $w \cdot\left|V_{R}\right|$ respectively) as inputs and returns a vector $\vec{z}$ (of size $\left|V_{R}\right|$ ) that shows which of $R$ 's values also belong to $V_{S}$. This function can be represented by a circuit of boolean gates. $S$ hardwires its input $\vec{x}$ into the circuit and supplements each possible encrypted bit value at each circuit wire with its own random key (used for decrypting the next gate's output and its key). The protocol has two major steps:

Coding $R$ 's input: For each bit of $\vec{y}, R$ engages with $S$ in a 1-out-of-2 oblivious transfer protocol $[36,39]$ and gets the corresponding supplemental keys.

Computing the circuit: For each gate, $R$ receives a table from $S$ and, using the keys for the gate's inputs, computes the output and its key. In the process, $R$ applies a pseudorandom function twice per each output wire.

To get $f(\vec{x}, \vec{y}), R$ gets the tables that allow it to decrypt the wires with the output of the circuit.

\section{A.1 Cost Analysis}

Let the keys (for the circuit gates) be $k_{0}$ bits long, and $C_{r}$ be the cost of pseudorandom function evaluation. We assume that $w=32$ (recall that $w$ is the size in bits of the input values), $k_{0}=64$, and $\left|V_{R}\right|=\left|V_{S}\right|=n$.

\section{A.1.1 Coding the Input}

Let $C_{\text {ot }}$ be the computation cost of each oblivious transfer, and $C_{\text {ot }}^{\prime}$ its communication cost. An efficient protocol for oblivious transfers is given in [36]. For any integer $l>0$, this paper gives a protocol with amortized cost

$$
C_{\mathrm{ot}}=\frac{1}{l} \cdot C_{e}+\frac{2^{l}}{l} \cdot C_{\times} ; \quad C_{\mathrm{ot}}^{\prime}>\frac{2^{l}}{l} \cdot k_{1}
$$

where $C_{\times}$is the cost of multiplication, and $k_{1}$ is the size of the keys used in oblivious transfer. We assume $k_{1}=100$ [36]. Assume that $C_{e}=1000 C_{\times}$; then the best choice with respect to the computation time is $l=8$, and the costs become

$$
C_{\mathrm{ot}}=0.157 C_{e} ; \quad C_{\mathrm{ot}}^{\prime}>32 k_{1} \text {. }
$$

Cost The computation cost of coding the input is

$$
w \cdot\left|V_{R}\right| \cdot C_{\mathrm{ot}}=32 \times n \times 0.157 C_{e} \approx 5 n C_{e}
$$

and the communication cost is

$$
w \cdot\left|V_{R}\right| \cdot C_{\mathrm{ot}}^{\prime}>32 \times n \times 32 k_{1} \approx 10^{5} n
$$

\section{A.1.2 Evaluating the Circuit}

Let $C\left(w,\left|V_{S}\right|,\left|V_{R}\right|\right)$ be the total number of gates required for the circuit. We estimate lower bounds on the number of gates required for a brute force algorithm, and a more efficient partitioning algorithm.

Let $G_{e}$ be the number of gates required to compare two $w$-bit numbers in the circuit to determine whether they are equal. Let $G_{l}$ be the number of gates required to determine which number is less than (or equal to) the other.

Brute Force Circuit Consider a circuit that compares every number in $V_{R}$ with every number in $V_{S}$, and then merges the results to 
output just the numbers in $V_{R}$ that were equal to at least one number in $V_{S}$. The number of gates $C\left(w,\left|V_{S}\right|,\left|V_{R}\right|\right)$ in this circuit is greater than

$$
\left|V_{R}\right| \cdot\left|V_{S}\right| \cdot G_{e}
$$

Partitioning Circuit We assume that each set $V_{R}$ and $V_{S}$ is given to the circuit in the form of an ordered array, with all duplicates removed. Instead of comparing all pairs of numbers, we can split these arrays into $m$ intervals (non-interleaving subarrays) of size $\left|V_{R}\right| / m$ and $\left|V_{S}\right| / m$. For ease of exposition, we assume that $\left|V_{R}\right|=\left|V_{S}\right|=n$, and that $n$ is a power of $m$.

Out of all possible $m^{2}$ pairs of subarrays, with one subarray from $V_{S}$ and the other from $V_{R}$, only at most $2 m-1$ pairs may have a nonempty intersection; the others are pairs of non-interleaving subarrays. To see this, note that in a pair of interleaving subarrays the beginning of one subarray must be within the interval spanned by the other. There is at most one pair per one such "internal beginning." There are $2 m$ subarrays in both $V_{S}$ and $V_{R}$, each having only one beginning; and the smallest beginning is always "wasted," thus limiting the number of interleaving pairs to $2 m-1$.

The circuit has to choose the $2 m-1$ interleaving pairs of subarrays and then use recursion to compute set intersections within these pairs. To check whether a pair of subarrays interleaves, we need to compare the smallest and largest numbers of these subarrays, thus making 2 comparisons. There are $m^{2}$ pairs, so we need $2 m^{2}$ comparisons, and hence $2 m^{2} G_{l}$ gates. Additional gates are needed to reroute the subarrays and combine the recursive outputs, but we shall ignore them in our estimation, since we are interested primarily in a lower bound for the cost of the circuit (using this algorithm).

Let $f(n)$ be the cost of the circuit. Then

$$
\begin{aligned}
& f(n)>2 m^{2} G_{l}+(2 m-1) \cdot f(n / m) \\
& f(1)=G_{e}
\end{aligned}
$$

Let $c=2 m^{2} G_{l}$; then

$$
\begin{aligned}
f(n) & >c+(2 m-1) \cdot f(n / m) \\
& >c+(2 m-1) \cdot\left(c+(2 m-1) f\left(n / m^{2}\right)\right) \\
& >c \cdot \sum_{i=0}^{\log _{m} n-1}(2 m-1)^{i}+(2 m-1)^{\log _{m} n} f(1) \\
& =c \cdot \frac{(2 m-1)^{\log _{m} n}-1}{(2 m-1)-1}+(2 m-1)^{\log _{m} n} G_{e} \\
& =c \cdot \frac{n^{\log _{m}(2 m-1)}-1}{2 m-2}+n^{\log _{m}(2 m-1)} G_{e} \\
& >\left(\frac{c}{2 m-2}+G_{e}\right) \cdot\left(n^{\log _{m}(2 m-1)}-1\right)
\end{aligned}
$$

Substituting back the value for $c$, we get

$$
f(n)>\left(\frac{m^{2}}{m-1} G_{l}+G_{e}\right) \cdot\left(n^{\log _{m}(2 m-1)}-1\right)
$$

Two $w$-bit numbers can be checked for equality using $2 w-1$ binary gates and compared using $5 w-3$ gates. Setting $G_{l}=5 w-3$ and $G_{e}=2 w-1$ gives

$$
f(n)>\left(\frac{m^{2}}{m-1}(5 w-3)+(2 w-1)\right) \cdot\left(n^{\log _{m}(2 m-1)}-1\right)
$$

Brute Force vs. Partitioning Let $\left|V_{R}\right|=\left|V_{S}\right|=n$. (As before $w=32$ and $k_{0}=64$.) Then, for the partitioning circuit, we get the following values for $C\left(w,\left|V_{R}\right|,\left|V_{S}\right|\right)=f(n)$ for the optimal value of $m$ :

\begin{tabular}{|ccc|}
\hline$n$ & $m$ & $f(n)$ \\
\hline 10,000 & 11 & $2.3 \times 10^{8}$ \\
1 million & 19 & $7.3 \times 10^{10}$ \\
100 million & 32 & $1.9 \times 10^{13}$ \\
\hline
\end{tabular}

The brute force circuit does much worse, with $C\left(w,\left|V_{R}\right|,\left|V_{S}\right|\right)$ equal to $6.3 \times 10^{9}, 6.3 \times 10^{13}$, and $6.3 \times 10^{17}$ respectively.

Cost For each gate in the circuit, $R$ gets a table from $S$ whose size is $4 k_{0}$, and evaluates 2 pseudorandom functions. Therefore the computation cost of circuit evaluation is

$$
2 C_{r} \cdot C\left(w,\left|V_{S}\right|,\left|V_{R}\right|\right)=2 C_{r} f(n)
$$

and the communication cost is

$$
4 k_{0} \cdot C\left(w,\left|V_{S}\right|,\left|V_{R}\right|\right)=256 \cdot f(n) .
$$

\section{A.2 Comparison with Our Protocol}

Computation We get the following computation costs:

\begin{tabular}{|c|c|c|c|}
\hline$n$ & \multicolumn{2}{|c|}{ Circuit } & \multirow{2}{*}{ Our Protocol } \\
\cline { 2 - 3 } & Input (OT) & Evaluation & \\
\hline $10^{4}$ & $5 \times 10^{4} C_{e}$ & $4.7 \times 10^{8} C_{r}$ & $4 \times 10^{4} C_{e}$ \\
$10^{6}$ & $5 \times 10^{6} C_{e}$ & $1.5 \times 10^{11} C_{r}$ & $4 \times 10^{6} C_{e}$ \\
$10^{8}$ & $5 \times 10^{8} C_{e}$ & $3.8 \times 10^{13} C_{r}$ & $4 \times 10^{8} C_{e}$ \\
\hline
\end{tabular}

The cost of coding the input for the circuit is slightly higher than the cost of our protocol. The total cost of the circuit (relative to our protocol) depends on the ratio of $C_{e}$ to $C_{r}$. While $C_{e} \gg C_{r}$, there are $10^{4}$ to $10^{5}$ as many calls to $C_{r}$ as there are to $C_{e}$. Thus our protocol will be substantially faster if $C_{r}>C_{e} / 10000$, and slightly faster otherwise.

Communication The communication costs (in bits) are:

\begin{tabular}{|c|c|c|c|}
\hline$n$ & \multicolumn{2}{|c|}{ Circuit } & \multirow{2}{*}{ Our Protocol } \\
\cline { 2 - 3 } & Input (OT) & Circuit (Tables) & \\
\hline $10^{4}$ & $10^{9}$ & $6.0 \times 10^{10}$ & $3 \times 10^{7}$ \\
$10^{6}$ & $10^{11}$ & $1.8 \times 10^{13}$ & $3 \times 10^{9}$ \\
$10^{8}$ & $10^{13}$ & $4.9 \times 10^{15}$ & $3 \times 10^{11}$ \\
\hline
\end{tabular}

The circuit-based protocol requires 1000 to 10,000 times as much communication as our protocol. For $n=1$ million, the communication time for the circuit-based protocol is 144 days (using a T1 line), versus 0.5 hours for our protocol. The communication cost makes the circuit-based protocol impractical for database-size applications. 